\title{
Micropuntura com radiofrequência no tratamento da ceratopatia bolhosa sintomática
}

\author{
Micropuncture with radiofrequency for the treatment \\ of symptomatic bullous keratopathy
}

Adriana Carneiro Macedo¹, Cristiane Ishirara², Daniella Fairbanks³, Vicente Vitiello Neto ${ }^{4}$, José Ricardo Carvalho Lima Rehder ${ }^{5}$

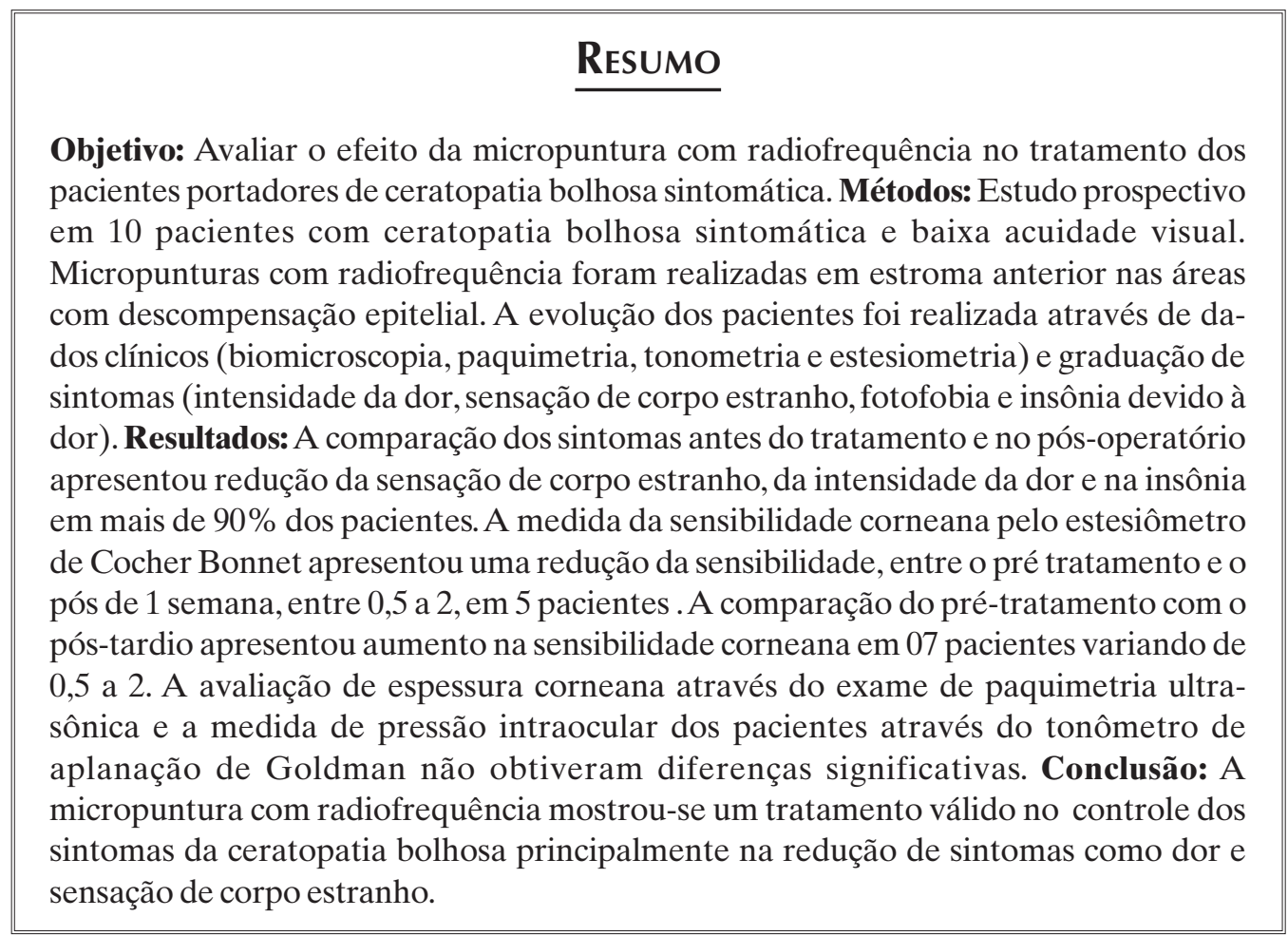

Descritores: Doenças da córnea/cirurgia; Edema da córnea; Lâmina limitante posterior da córnea/cirurgia

\footnotetext{
${ }^{1}$ Estagiário no Setor Córnea e de Patologia Externa da Disciplina de Oftalmologia da Faculdade de Medicina do ABC - FMABC Santo André (SP), Brasil;

${ }^{2}$ Colaboradora no Setor Córnea e de Patologia Externa da Disciplina de Oftalmologia da Faculdade de Medicina do ABC - FMABC Santo André (SP), Brasil;

${ }^{3}$ Colaboradora no Setor de Córnea e Patologia Externa da Disciplina de Oftalmologia da Faculdade de Medicina do ABC - FMABC Santo André (SP), Brasil;

${ }^{4}$ Chefe do Setor de Córnea e Patologia Externa da Disciplina de Oftalmologia da Faculdade de Medicina do ABC - FMABC - Santo André (SP), Brasil;

${ }^{5}$ Professor Titular da Disciplina de Oftalmologia da Faculdade de Medicina do ABC - FMABC - Santo André (SP), Brasil.

Recebido para publicação em: 22/9/2009 - Aceito para publicação em 26/11/2009
} 

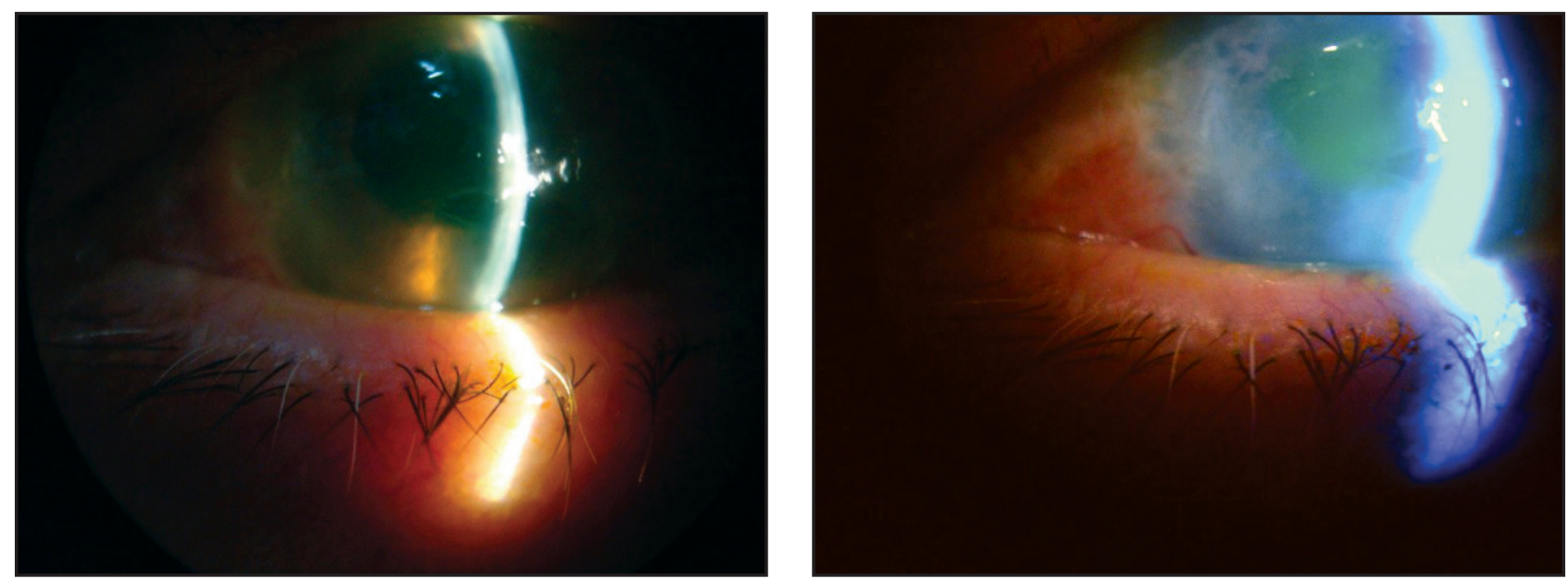

Figura 1: Imagem de biomicroscopia de córnea com bolha epitelial sem e com corante de fluoresceína

\section{INTRODUÇÃO}

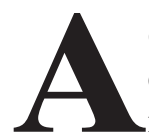

ceratopatia bolhosa é uma doença corneana causada por descompensação endotelial, com

isso não há deturgescência adequada do estroma corneano, propiciando a formação de edema estromal com formação de bolhas epiteliais e subepiteliais ${ }^{(1-6)}$. Essas, quando rompem expõem terminações nervosas causando dor, fotofobia e lacrimejamento ${ }^{(1-45)}$. Os pacientes frequentemente apresentam diminuição da acuidade visual, fotofobia, epífora e um aumento do risco de ceratite infecciosa ${ }^{(3)}$. São causas de ceratopatia bolhosa: trauma, glaucoma e anormalidades congênitas ${ }^{(2-3-6-8)}$.

Sem função endotelial adequada, há a hidratação do estroma corneano com perda dos ceratócitos e atenuação mais rompimento da camada de Bowman e membrana basal. Eventualmente há perda de glicosaminoglicans no estroma ${ }^{(9)}$. Esses fatores levam a mudanças na superfície ocular com perda de adesividade epitelial evoluindo com erosão recorrente e depois persistente.

Enquanto a ceratoplastia penetrante ou a ceratoplastia lamelar posterior ${ }^{(4)}$ são os tratamentos definitivos para a ceratopatia bolhosa, existem alternativas terapêuticas com resultados variáveis que podem ser utilizadas no controle dos sintomas em pacientes sem potencial de recuperação visual ou naqueles sintomáticos que aguardam na fila de transplante de córnea ${ }^{(6)}$.

Vários tratamentos são relatados na literatura. Colírios antiglaucomatosos e hiperosmóticos como o $\mathrm{NaCl} 5 \%$ e dimetilpolisiloxane para tentar diminuir o edema epitelial e estromal, uso de lente de contato terapêutica (LTC), ceratotomia anular, epiceratoplastia, ceratectomia fototerapêutica por excimer laser (PTK),

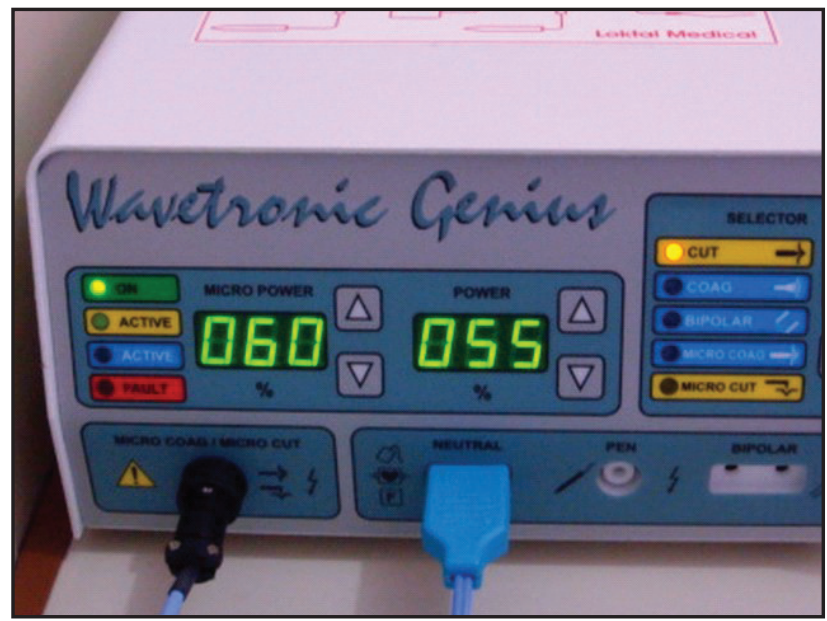

Figura 2: Aparelho de radiofrequência usado no procedimento de micropunturas e parâmetros utilizados

recobrimento conjuntival ${ }^{(1)}$ e com membrana amniótica ${ }^{(5)}$, criopexia (cauterização) e punção do estroma anterior (PEA-micropunctura) ${ }^{(1-8-10)}$ com agulha de insulina.

Em 1996, Cormier et al. relataram uma diminuição na formação de bolhas e alívio da dor em pacientes com ceratopatia bolhosa submetidos à puntura do estroma anterior ${ }^{(1-3)}$. Com a micropuntura há destruição da camada de Bowman, induzindo fibrose e melhor adesão do epitélio ao estroma subjacente ${ }^{(1,9,11,12)}$.

A termoceratoplastia com radiofrequência (ceratoplastia condutiva) é um procedimento cirúrgico aprovado pelo FDA, em 2002, para fins refrativos. A radiofrequência eleva a temperatura no estroma corneano, provocando um encolhimento das fibras de colágeno de forma duradoura, comprovado em pesquisas microscópicas ${ }^{(6,10,13-15)}$.

O presente estudo tem por objetivo avaliar o efeito 

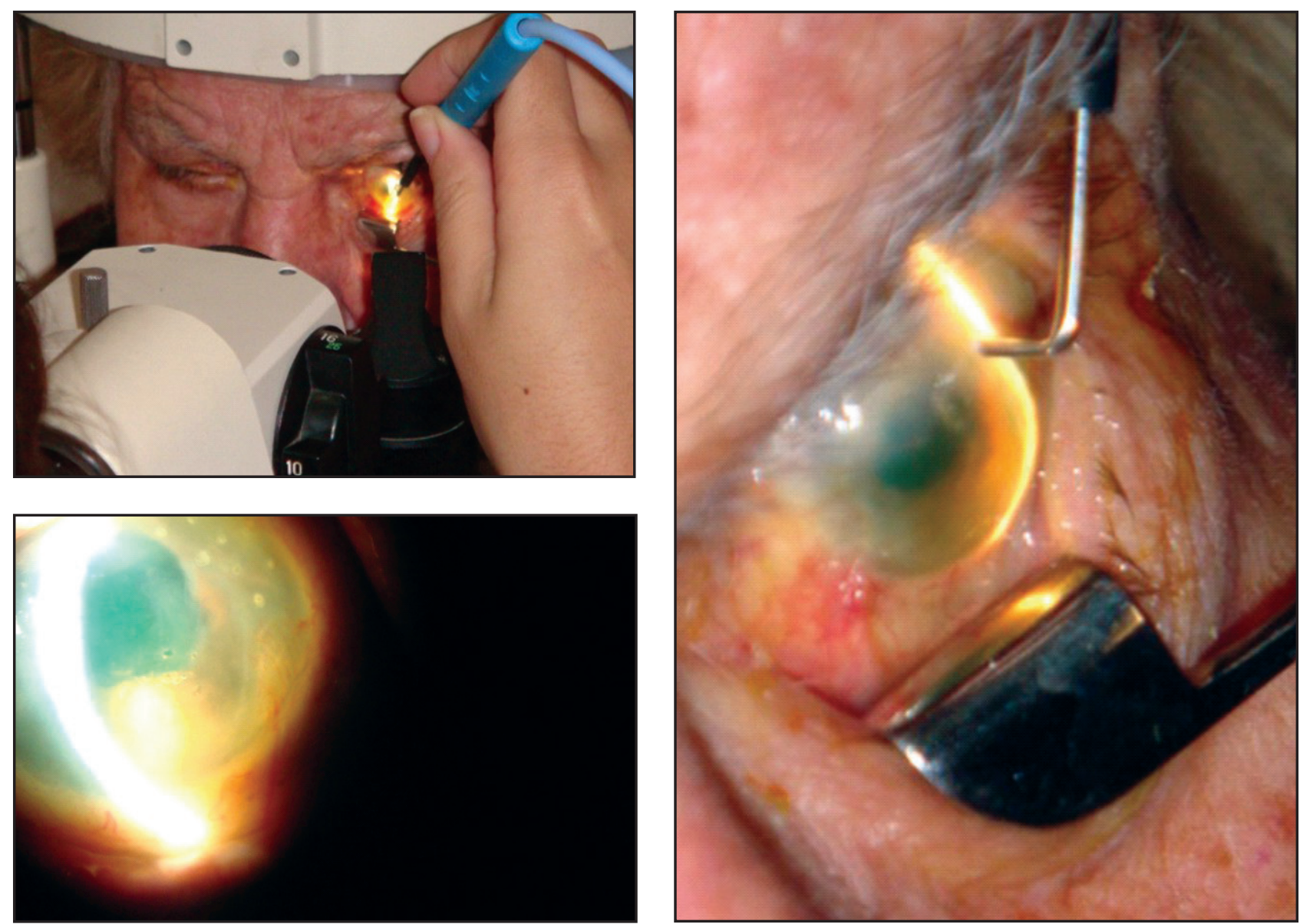

Figura 3: Aplicação da radiofrequência pela ponteira agulhada em região corneana na lâmpada de fenda e as cicatrizes corneanas observadas no seguimento pós-micropuntura por radiofrequência

da micropuntura com radiofrequência no alívio da dor e melhora dos defeitos epiteliais recorrentes em pacientes portadores de ceratopatia bolhosa sintomática e pobre acuidade visual.

\section{Métodos}

Após aprovação pelo Comitê de Ética e assinado Termo de Ciência e Consentimento, foi realizado estudo prospectivo em 10 pacientes, acompanhados no setor de Córnea e Patologia Externa da Disciplina de Oftalmologia da Faculdade de Medicina do ABC,no período de novembro de 2007 a julho de 2008.

Os critérios de inclusão no estudo foram ceratopatia bolhosa descompensada (figura 1) e sintomática e melhor acuidade visual $\leq 20 / 400$.

A avaliação oftalmológica incluiu medida da acuidade visual, biomicroscopia à lâmpada de fenda, estesiometria (estesiômetro de Cochet - Bonnet), paquimetria e medida de pressão intraocular. A mensuração dos sintomas foi realizada através de questionário aplicado aos pacientes durante cada retorno, sendo solicitada a graduação dos sintomas por questionário subjetivo numa escala de 0 a 3 , e presença de insônia relacionada ao quadro doloroso. Os itens que constaram na avaliação foram os seguintes: sensação de corpo estranho, intensidade da dor, fotofobia.

A micropuntura com radiofrequência foi realizada por dois fellows do serviço de córnea treinados para o procedimento. $\mathrm{O}$ mesmo foi realizado à lâmpada de fenda, sob anestesia tópica com colírio anestésico, colocação de blefarostato ligado ao aparelho de radiofrequência (Wavetronic) Loktal ${ }^{\circledR}$, ajustado a potência em $60 \mathrm{~mJ}$ no micropower e $55 \mathrm{~mJ}$ no power (figura 2). As perfurações foram realizadas no estroma anterior com ponteira reta, perpendicular ao plano da córnea, apenas nas áreas com evidências de bolhas, inclusive em região central de córnea (figura 3). Após o procedimento foi colocada lente de contato terapêutica e entre- 
Tabela 1

Perfil epidemiológico e clínico dos pacientes avaliados no estudo

\begin{tabular}{ccccccc}
\hline Paciente & Idade & Sexo & Olho acometido & $\begin{array}{c}\text { Condição ocular } \\
\text { precipitante }\end{array}$ & $\begin{array}{c}\text { Patologia ocular } \\
\text { associada }\end{array}$ & $\begin{array}{c}\text { Intervalo entre início } \\
\text { dos sintomas e aplicação } \\
\text { da micropuntura (meses) }\end{array}$ \\
\hline 1 & 71 & Feminino & OE & ICE síndrome & Glaucoma & 16 \\
2 & 73 & Feminino & OE & Pseudofácica & - & 28 \\
3 & 75 & Masculino & OD & Afácica & - & 61 \\
4 & 77 & Feminino & OE & Pseudofácica & - & 19 \\
5 & 79 & Feminino & OE & Afácica & - & 36 \\
6 & 43 & Masculino & OD & Pseudofácica & - & 11 \\
7 & 79 & Masculino & OD & Pseudofácica & - & 12 \\
8 & 88 & Feminino & OD & Pseudofácica & Glaucoma & \\
9 & 79 & Feminino & OD & Pseudofácica & - & - \\
10 & 70 & Feminino & OE & Pseudofácica & & - \\
\hline
\end{tabular}

Tabela 2

Dados do exame oftalmológico

\begin{tabular}{|c|c|c|c|c|c|c|c|c|c|c|}
\hline \multirow[t]{2}{*}{ Pacientes } & \multirow{2}{*}{$\begin{array}{c}\text { Olho } \\
\text { acometido }\end{array}$} & \multicolumn{3}{|c|}{ Acuidade visual } & \multicolumn{3}{|c|}{ Paquimetria (média) } & \multicolumn{3}{|c|}{ Pressão intraocular } \\
\hline & & Pré & $\begin{array}{l}\text { Pós } 1 \\
\text { sem }\end{array}$ & $\begin{array}{l}\text { Pós- } \\
\text { tardio }\end{array}$ & Pré & $\begin{array}{l}\text { Pós } \\
1 \text { sem }\end{array}$ & $\begin{array}{c}\text { Pós- } \\
\text { tardio }\end{array}$ & Pré & $\begin{array}{l}\text { Pós } \\
1 \text { sem }\end{array}$ & $\begin{array}{l}\text { Pós } \\
\text { tardio }\end{array}$ \\
\hline 1 & $\mathrm{OE}$ & MM & CD1m & $\mathrm{CD} 40 \mathrm{~cm}$ & 759 & 720 & 752 & 14 & 12 & Mira irreg \\
\hline 2 & $\mathrm{OE}$ & CD50cm & $\mathrm{CD} 1 \mathrm{~m}$ & $\mathrm{CD} 1 \mathrm{~m}$ & 761 & - & 669 & 14 & Mira irreg & 12 \\
\hline 3 & OD & MM & $\mathrm{MM}$ & $\mathrm{MM}$ & 833 & - & - & 12 & 12 & Mira irreg \\
\hline 4 & $\mathrm{OE}$ & MM & MM & $\mathrm{CD} 15 \mathrm{~cm}$ & 848 & - & 683 & Mira irreg & 10 & 13 \\
\hline 5 & $\mathrm{OE}$ & MM & MM & MM & 814 & 903 & 909 & 12 & 10 & 12 \\
\hline 6 & OD & MM & $\mathrm{MM}$ & MM & 616 & 754 & 609 & 15 & 16 & 12 \\
\hline 7 & OD & CD1m & $\mathrm{CD} 50 \mathrm{~cm}$ & $\mathrm{CD} 40 \mathrm{~cm}$ & 770 & 799 & 791 & 12 & 08 & 10 \\
\hline 8 & OD & MM & $\mathrm{MM}$ & MM & - & - & 843 & 12 & 10 & 07 \\
\hline 9 & OD & MM & PL & $\mathrm{MM}$ & - & - & 916 & 10 & 08 & 10 \\
\hline 10 & $\mathrm{OE}$ & CD $30 \mathrm{~cm}$ & $\mathrm{CD} 30 \mathrm{~cm}$ & $\mathrm{CD} 1 \mathrm{~m}$ & 556 & 541 & 703 & 10 & 10 & 13 \\
\hline
\end{tabular}

Tabela 3

Medidas de sensibilidade cornena estesiômetro de Cochet Bonnet

\begin{tabular}{cccc}
\hline Pacientes & \multicolumn{3}{c}{ Estesiometria } \\
\cline { 2 - 4 } & Pré & Pós 1 sem & Pós-tardio \\
\hline 1 & 2 & 1,5 & 3 \\
2 & 2 & 0 & 1,5 \\
3 & 1,5 & - & 2 \\
4 & 1,5 & 1,0 & 2 \\
5 & 2 & 3 & 3,5 \\
6 & 4 & 5 & 6 \\
7 & 2,5 & 1,5 & 2 \\
8 & 2,5 & 1,5 & 2,0 \\
9 & 0 & 0 & 2 \\
10 & 3,5 & - & 4 \\
\hline
\end{tabular}



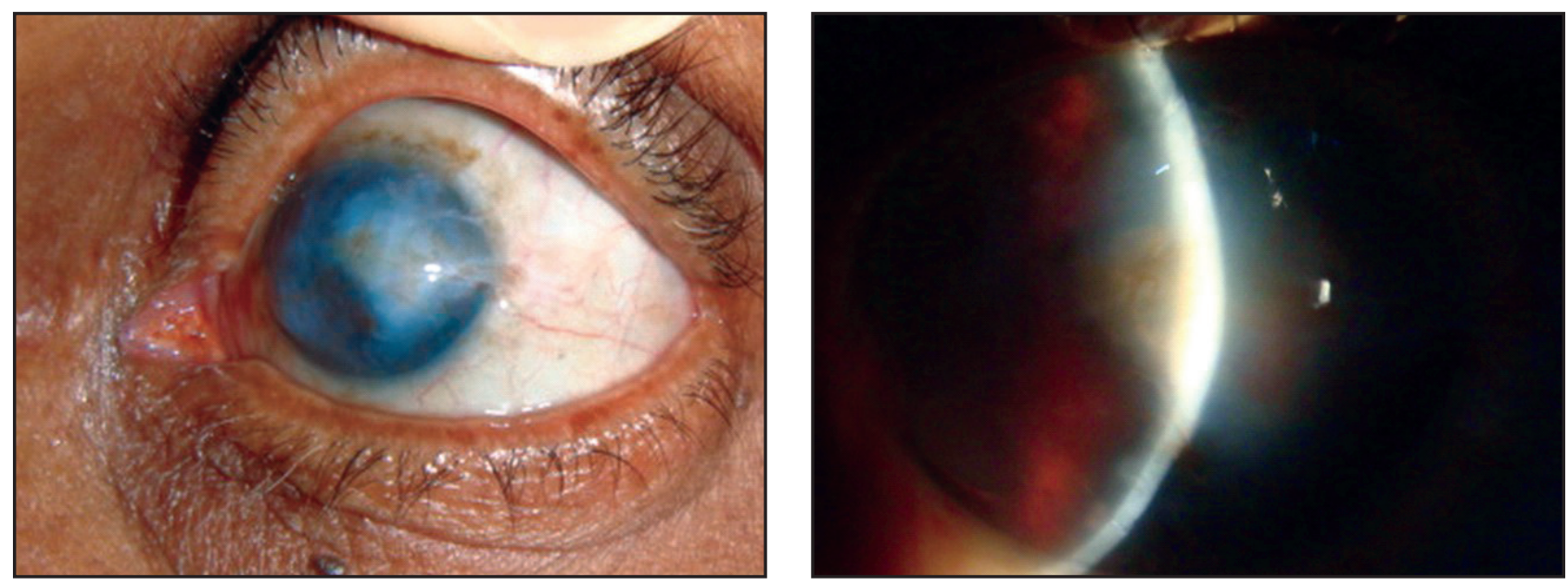

Figura 4: Seguimento tardio de paciente com afinamento corneano pós-micropuntura

Tabela 4

Relação e graduação subjetiva dos sintomas pelos pacientes

\begin{tabular}{|c|c|c|c|c|c|c|c|c|c|c|c|c|c|}
\hline \multirow[t]{2}{*}{ Pact } & \multicolumn{3}{|c|}{ Sensação de CE } & \multicolumn{3}{|c|}{ Intensidade da dor } & \multicolumn{3}{|c|}{ Fotofobia } & & \multicolumn{3}{|c|}{ Insônia por dor } \\
\hline & Pré & $\begin{array}{l}\text { Pós } \\
1 \text { sem }\end{array}$ & $\begin{array}{l}\text { Pós- } \\
\text { tardio }\end{array}$ & Pré & $\begin{array}{l}\text { Pós } \\
1 \text { sem }\end{array}$ & $\begin{array}{l}\text { Pós- } \\
\text { tardio }\end{array}$ & Pré & $\begin{array}{l}\text { Pós } \\
1 \text { sem }\end{array}$ & $\begin{array}{l}\text { Pós- } \\
\text { tardio }\end{array}$ & & Pré & $\begin{array}{l}\text { Pós } \\
1 \text { sem }\end{array}$ & $\begin{array}{c}\text { Pós } \\
\text { tardio }\end{array}$ \\
\hline 1 & 3 & 1 & 0 & 3 & 0 & 0 & 3 & 0 & 3 & & 2 & 0 & 0 \\
\hline 2 & 3 & 3 & 0 & 3 & 3 & 0 & 3 & 2 & 0 & & 3 & 3 & 0 \\
\hline 3 & 2 & 1 & - & 3 & 2 & - & 3 & 2 & - & & 3 & 0 & - \\
\hline 4 & 3 & 3 & 0 & 3 & 3 & 0 & 3 & 3 & 1 & & 3 & 0 & 0 \\
\hline 5 & 3 & 1 & 0 & 3 & 0 & 0 & 3 & 1 & 1 & 5 & 3 & 0 & 0 \\
\hline 6 & 0 & 0 & 0 & 3 & 1 & 0 & 2 & 2 & 0 & & 0 & 0 & 0 \\
\hline 7 & 3 & 3 & 0 & 2 & 1 & 0 & 3 & 3 & 0 & & 0 & 0 & 0 \\
\hline 8 & 3 & 0 & 0 & 3 & 0 & 0 & 3 & 0 & 0 & & 3 & 0 & 0 \\
\hline 9 & 0 & 0 & 0 & 0 & 0 & 0 & 2 & 0 & 0 & & 0 & 0 & 0 \\
\hline 1 & 02 & 1 & 1 & 2 & 0 & 0 & 3 & 1 & 0 & & 0 & 0 & 0 \\
\hline
\end{tabular}

gue medicação tópica, Ofloxacino 4x/dia e Diclofenaco sódico colírio $3 \mathrm{x} / \mathrm{dia}$, para ser instilado pelo paciente durante 7 e 3 dias, respectivamente.

Os retornos foram agendados para 1 semana após o procedimento, inicialmente, e nas semanas subsequentes até regressão importante dos sintomas.

\section{Resultados}

Foram avaliados 10 olhos ( 5 olhos direito e 5 olhos esquerdo) de 10 pacientes. Sete eram do sexo feminino e 3 do sexo masculino. A idade dos indivíduos variou de 43 a 79 anos, média de 73,4 anos (tabela 1).

A patologia ocular precipitadora do quadro de ceratopatia bolhosa foi trauma cirúrgico (pós-cirurgia de catarata) em 9 pacientes $(90 \%)$ sendo, no mo- mento do estudo, 7 pseudofácicos e 2 afácicos; e em 1 paciente $(10 \%)$ secundário à síndrome iridocorneana endotelial (ICE síndrome). A presença de doença ocular associada foi diagnosticado em 2 pacientes, ambos com glaucoma. O intervalo entre o início dos sintomas e a aplicação da micropuntura com radiofrequência variou de 11 meses a 61 meses, média de 24,3 meses (tabela 1).

A aplicação da micropuntura com radiofrequência foi realizada nas regiões da córnea com evidência de bolha, inclusive região central, sendo necessárias entre 1 a 2 sessões (com intervalo de pelo menos 1 semana). O número de consultas variou de 3 a 10 (acompanhamento de 02 a 07 meses - média 3.4 meses)

A melhor acuidade visual desses pacientes na avaliação pré-procedimento de micropuntura e após, no acompanhamento, encontra-se descrita na tabela 2 . 
A avaliação de espessura corneana através do exame de paquimetria ultrassônica não obteve todas as leituras em alguns pacientes, tanto na visita inicial, como no seguimento. Na visita inicial, os valores paquimétricos variaram de 616 a 848 (média de 744,6) e na visita final de 609 a 916 (média de 771,5). Nos pacientes onde foi possível avaliar a diferença entre o pré e pós, houve aumento da espessura corneana em 2 pacientes (com variação média de $58 \mu \mathrm{m}$ ), diminuição da espessura em 4 pacientes (com variação média de $67,7 \mu \mathrm{m})$. Em 2 pacientes o aparelho só conseguiu realizar leitura no pós- tardio, e em 1 , apenas no pré-procedimento (tabela 2 ).

A medida de pressão intraocular dos pacientes através do tonômetro de aplanação de Goldman também apresentou limitações de uso por irregularidade de superfície; A pressão média no pré e no pós-procedimento foi de 11,1. A variação de pressão no período estudado foi no máximo de $\pm 5 \mathrm{mmHg}$ (tabela 2).

Nos pacientes onde foi realizada a medida da sensibilidade corneana pelo estesiômetro de Cochet Bonnet, 5 pacientes apresentaram uma redução da sensibilidade, entre o pré-tratamento e o pós de 1 semana, entre 0,5 a 2 (média 1) e 2 pacientes apresentaram um aumento da sensibilidade de 1 no mesmo período. A comparação do pré-tratamento com o pós-tardio apresentou aumento na sensibilidade corneana em 07 pacientes variando de 0,5 a 2 (média 1,1) e redução de 0,5 em 3 pacientes (tabela 3).

A tabela 4 apresenta os dados sobre a avaliação subjetiva dos pacientes pela graduação dos sintomas quanto a sensação de corpo estranho, intensidade da dor, fotofobia e insônia por dor. A insônia por dor foi referida como grau 3 por 5 pacientes $(50 \%)$ no pré-tratamento, por 1 paciente no pós 1 semana e graduado em zero por $100 \%$ dos pacientes no pós-tardio. (Tabela 4).

A recuperação após o procedimento na maioria nos pacientes ocorreu sem intercorrências, com retirada da lente terapêutica no máximo em 15 dias, mantendo cicatrizes no local da micropuntura (figura 3). No paciente 4 , foi observado afinamento corneano progressivo em região paracentral temporal inferior a $2 \mathrm{~mm}, 2$ meses após a aplicação das micropunturas por radiofrequência. Foi realizado tratamento com cola de cianoacrilato e lente de contato terapêutica associado à medicação tópica. O mesmo manteve progressão do afinamento, sendo necessário recobrimento conjuntival da área corneana alterada 15 dias após a tentativa com a cola. Havendo resolução completa do quadro (figura 4 ).

\section{Discussão}

A descompensação endotelial secundária se deve a diferentes causas, mais comumente trauma cirúrgico ou não, inflamação, glaucoma e distrofia de Fuchs cursa com edema estromal e bolhas epiteliais. Os sintomas relacionados ao quadro de ceratopatia bolhosa, devido a sua intensidade, interferem com a qualidade de vida dos pacientes ${ }^{(2,11)}$.

Várias modalidades terapêuticas, descritas anteriormente, são utilizadas para alívio dos sintomas. Além do custo com a manutenção do tratamento, há aumento do risco de neovascularização corneana e infecção, no caso das lentes de contato para fins terapêuticos, ou aumento de inflamação, como no uso crônico de medicações tópicas.

Apesar do tratamento de escolha ser a ceratopatia penetrante, alguns tratamentos de punturas no estroma anterior da córnea tem se mostrado efetivo no controle dos sintomas relacionados ao despreendimento epitelial, por estimular a produção de proteínas na matrix extracelular e induzir o desenvolvimento de fibroses subepiteliais, evitando descolamento, como descrito por Cornier et al. ${ }^{(1)}$.

A micropuntura com radiofrequência, além da ação mecânica de punção no epitélio, estimulando a reação de fibroblastos, provê uma ação térmica, permitindo encolhimento das fibras de colágeno ${ }^{(6,14)}$.

Os pacientes acompanhados nesse estudo não apresentaram padrão de resposta significante quanto aos dados paquimétricos pós-procedimento, havendo desde o aumento da espessura quanto a redução. A irregularidade corneana, dificultando leituras, e a variação individual de resposta à produção de proteínas e colágeno podem ocasionar essas variações.

A aferição tonométrica também foi dificultada pela irregularidade epitelial associada ao quadro de ceratopatia bolhosa, pois as miras, observadas no tonômetro de aplanação de Goldman, tornavam-se muito irregulares para permitir leitura confiável.

A estesiometria mostrou diminuição inicial da sensibilidade na primeira semana de acompanhamento, com retorno da sensibilidade no pós-procedimento tardio, possivelmente por reorganização dos nervos sensitivos corneanos após a injúria termomecânica. O que não foi acompanhado por aumento nos sintomas de desconforto, mostraram em mais de $90 \%$ dos pacientes redução da sensação de corpo estranho e da dor. A fotofobia se manteve sendo referida como queixa por $40 \%$ dos pacientes, mas em menor intensidade que o pré tratamento.

A intercorrência ocorrida com um dos pacientes durante o acompanhamento, cursando com afinamento corneano,já foi relatada na literatura como possível compli- 
cação no procedimento ${ }^{(1)}$, podendo ter sido desencadeada por maior número de punturas na área tratada, havendo necessidade de sempre preservar a área livre de puntura, como pela própria conduta medicamentosa adotada pelo paciente, apesar das orientações médicas, com permanência do uso tópico de antiinflamatório por 4 semanas.

Os pacientes acompanhados nesse estudo diminuíram a necessidade de instilação frequente de colírios lubrificantes e o uso de lente de contato terapêutica.

A micropuntura com radiofrequência mostrou-se um tratamento válido na ceratopatia bolhosa para a redução de sintomas como dor e sensação de corpo estranho. Tornou-se vantajoso quando comparado com a micropuntura com agulha, devido à menor necessidade de reaplicação. A desvantagem está no custo do equipamento e a necessidade de esterilização a cada uso.

A segurança do procedimento depende não apenas de uma técnica rigorosa, mas do acompanhamento próximo dos pacientes nas primeiras semanas.

Novos estudos comparativos são necessários para afirmar a eficácia do procedimento a longo prazo.

\section{Abstract}

Purpose: Evaluate the effect of micro puncture with radiofrequency as treatment of symptomatic patients with bullous keratopathy. Methods: Prospective study in 10 pacients with syntomatic bullous keratopathy and low vision. Micro puncture with radiofrequency was made in areas of the anterior stromal that had an epithelial descompensation. The evaluation of the patients was realized trough clinical examinations (biomicroscopy, pachymetry, tonometry and esthesiometry) and symptom graduation (pain intensity, foreign body sensation, photophobia and insomnia caused by pain). Results: The comparisons of symptoms before treatment and weeks after procedure showed a decrease, in $90 \%$ of patients, of the pain, foreign body sensation and insomnia. The measure of corneal sensibility by the Cocher Bonnet esthesiometer presents a reduction in corneal sensibility, among before and after one week of treatment, between 0,5 to 2, in 5 patients. But several weeks after treatment, the corneal sensibility aggrandized in 7 patients, between 0,5 to 2. The corneal thickness evaluation using ultra sonic pachymeter and Goldman tonometer not find important differences. Conclusion: The micro puncture with radiofrequency is a valid treatment in order to reduce symptoms in bullous keratopathy.

Keywords: Corneal diseases/surgery; Corneal edema; Corneal stroma; Descemet membrane/surgery

\section{REFERÊNCIAS}

1. Cormier G, Brunette I, Boisjoly HM, LeFrancois M, Shi ZH, Guertin MC. Anterior stromal punctures for bullous keratopathy. Arch Ophthalmol. 1996; 114 (6): 654-8.

2. Gomes JA, Haraguchi DK, Zambrano DU, Izquierdo Junior L, Cunha MC, de Freitas D. Anterior stromal puncture in the treatment of bullous keratopathy: six-month follow-up. Cornea. 2001; 20(6): 570-2.

3. Tsai TC, Su CY, Lin CP. Anterior stromal puncture for bullous keratophaty. Ophtahlmic Surgery, Lasers \& Imaging. 2003; 34(5):371-4.

4. Busin M, Arffa RG, Sebastiani A. Endokeratoplasty as an alternative to penetrating keratoplasdty for the surgical treatment of diseased endothelium: inicial results. Ophthalmology. 2000;107(11):2077-82.

5. Pires RTF, Tseng SC, Prabhasawat P, Puangrsricharem V, Maskin ST, Kim JC. Amniotic membrane transplantation for symptomatic bullous keratopathy. Arch Ophtalmol.1999; 117(10):1291-7.

6. Gasset AR, Kaufman HE. Thermokeratoplasty. Trans AM Acad Ophthalmol Otoryngol. 1973;7(4):OP441-54.

7. Malecha IMA. Anterior stromal puncture for recurrent corneal erosion after laser in situ keratomileusis. J Cataract Refract Surg. 2005; 31 (1):9-10.

8. Martins EN, Alvarenga LS, Sousa LB, Orlando filho VT, Gomes JA, de Freitas D. anterior stromal puncture in Brown-McLean syndrome. J Cataract Refract Surg. 2004; 30(7):1575-7.

9. Castellano AGD, Bardal AMC, Lago C, Moreira LB, Moreira H. Transplante de membrana amniótica na ceratopatia bolhosa. Arq Bras Oftalmol. 2004;67(6):855-8.

10. Hashizume N, Saika S, Ooshima A, Yamanaka O, Okada Y, Ohnishi Y. Corneal epithelial basement membrane after experimental anterior estromal puncture in guinea pigs: immunohistochemical study. Jpn J Ophthalmol. 1997; 41(6):376-80.

11. Judge D, Payant J, Frase IS, Wood TO. Anterior stromal micropuncture electron microscopic changes in the rabbit cornea. Cornea. 1990;9(2):152-60.

12. Hsu JK, Rubinfeld RS, Barry P, Jester JV. Anterior stromal puncture. Immunohistochemical studies in human corneas. Arch Ophthalmol. 1993; 111(8):1057-63.

13. Geggel HS, Maza CE. Anterior stromal puncture with the $\mathrm{Nd}$ :YAG laser. Invest Ophthalmol Vis Sci. 1990;31(8):1555-9.

14. Paulino LV, Paulino E, Barros RA, Salles AG, Rehder JRCL. Alteração topográfica da curvatura corneana após aplicação de radiofreqüência em modelo animal. Arq Bras Oftalmol. 2005;68(4):451-6.

15. Sridhar MS, Vemuganti GK, Bansal AK, Rao GN. Anterior Stromal puncture in bullous keratopathy: a clinicopathologic study. Cornea. 2001; 20(6): 573-9.

\section{Endereço para correspondência: \\ Rua Daniel de Moraes, $\mathbf{n}^{\circ} \mathbf{8 0}$ \\ Edf Residência Menlia - apto. 52 - Enseada \\ CEP 11440-330 - Guarujá - (SP), Brasil}

Email: adrimacedo@hotmail.com 